\title{
Power Saving Effect of Dynamic Partial Reconfiguration on FPGA
}

\author{
Satoshi Yonemitsu, Sota Kono, Akira Yamawaki* \\ Kyushu Institute of Technology, Kitakyushu, 804-8550 Japan \\ *Corresponding Author: yama@ecs.kyutech.ac.jp
}

\begin{abstract}
Dynamic Partial Reconfiguration (DPR) on Field Programmable Gate Array (FPGA) is able to update circuits equal to software without halting an entire system and achieve power saving by emptying unused circuits on the device. But, the way designing and managing circuit data of the DPR are dependent on each designer. Thus, the DPR has not become widespread because it's difficult to share the intellectual properties and execution models over different engineers. This paper defines the initial framework of the design and management methods for the DPR that the FPGA performs by itself, self DPR. We perform the case study based on the proposed framework. In addition, we indicates the effect of power saving depending on the circuit blanking using prototype.
\end{abstract}

Keywords: FPGA, DPR, design framework, management framework, power consumption

\section{Introduction}

The one of functions in the Field Programmable Gate Array (FPGA) which is able to change a hardware configuration after shipping is Dynamic Partial Reconfiguration (DPR). It can update circuits equal to software without halting an entire system. In addition, it brings some advantages such as cutting cost, making a board space small and power saving. At first, the DPR can execute a time - sharing processing because it changes the circuit in one part on the FPGA dynamically. In other words, it is able to implement using the FPGA with small capacity without large one in case of implementing a big scale circuit. It cuts costs consequently. Secondly, the DPR can convert circuit one after another in a specific part on the FPGA. So, designers don't have to implement multiple circuits at the same time and a board space is reduced. In addition, an internal circuit is removed by executing the circuit blanking for the unused part in multiple processing unit. It means that declining a toggle rate by suppressing an operation rate of nodes and we can save power consumption. Particularly these advantages are thought that contribute to the improvement of performance for small electronic devices.

The DPR however become widespread. One of reasons is how to design and manage circuit data on the occasion of operation depend on each designer. Thus, designers can't use the circuit data no matter what other designers create the wonderful one. Although there are large number of prior research using DPR technology for the data processing applications ${ }^{(1-3)}$, the researches about the unified framework of a design method and management of dynamic partial reconfiguration are few.

The purpose of this research is defining the framework of a design of self DPR that FPGA executes the DPR on itself, and management of circuit data on the occasion of operation. After that, we indicate the effect of power saving depending on the circuit blanking using prototype and trial system.

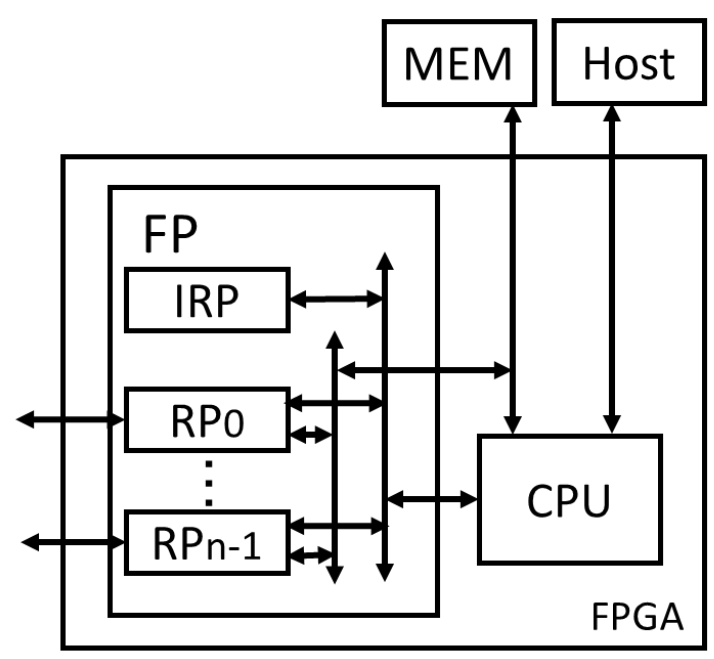

Fig. 1. Hardware framework 


\section{Design Framework of Self DPR}

\subsection{Outline of Hardware}

Fig. 1 shows an outline of hardware framework. CPU is an embedded processor on the FPGA, which manages the DPR and communicates with the host. Fixed Part (FP) is configured once when the system starts up and this part isn't reconfigured while the system is running. Reconfigurable parts (RPs) are reconfigured by the DPR while the system is running. The internal reconfigurable port (IRP) is the port to write circuit data to execute the DPR. The MEM is a general purpose memory to store reconfigurable circuit data, process data, management tables, and so on.

\subsection{Design Method of Circuit Data}

Fig. 2 shows the hierarchy among the fixed part and the reconfigurable parts at the hardware description language (HDL) level. The fixed part is a top module consisting of some reconfigurable parts. Each reconfigurable part has its own unified entity to communicate with the fixed part. The hardware modules in the reconfigurable part must have same entity even if the contents of the reconfigurable part are changed by the DPR.

Fig. 3 shows a design flow. The HDL files of the fixed part and the reconfigurable parts are synthesized into the netlists. The layout library has the layout data previously floor planned. Each layout data has the placement information of the partitions for the reconfigurable parts on the target physical hardware corresponding to Fig. 1. The designer select the dedicated layout data from the layout library and perform the automatic place and route with the netlists previously synthesized. Finally, the reconfigurable circuit data are generated. The reconfigurable data are those of the fixed part and the reconfigurable parts including the blank data.



Fig. 2. HDL hierarchy over fixed and reconfigurable part
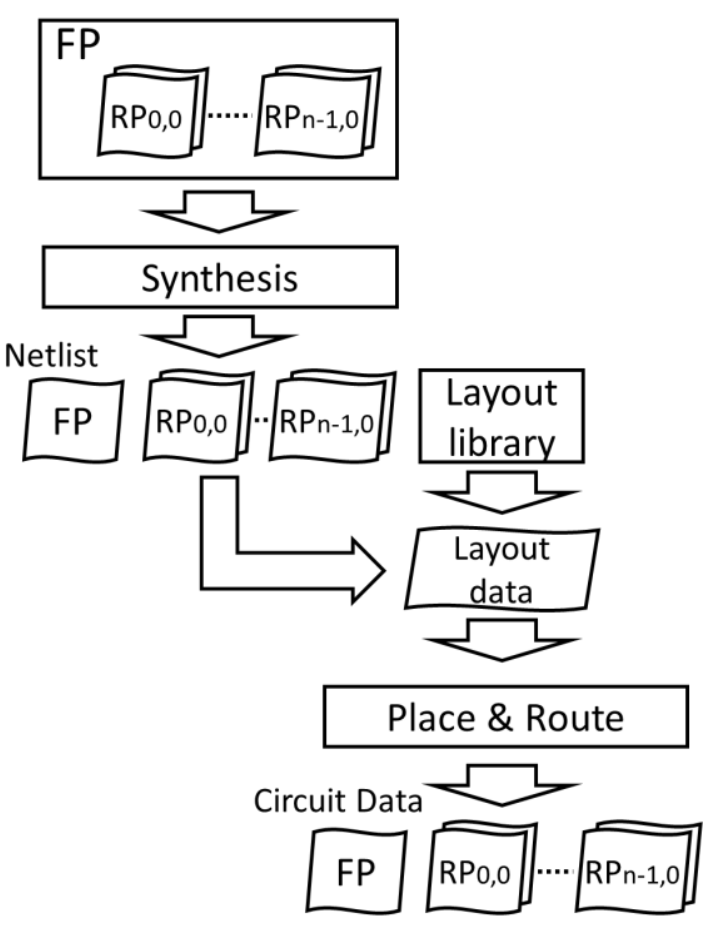

Fig. 3. Design flow

\section{Management Framework of Self DPR}

Fig. 4 shows the concept of the management framework of self DPR. Each functions needed by self DPR in this management framework are executed by entering commands from the host. Table 1 shows commands, functions and input data. "Reg_circ" registers circuit data to a memory through the FPGA embedded processor. Users enter the circuit ID with circuit data in this command. The FPGA embedded processor gets the head address of registered circuit data in a memory and file size of circuit data. Then the embedded processor preserves them, including the circuit ID, as the group. "Exe_circ" is the command to read corresponding circuit data from a memory by entering ID, after that it executes self DPR by writing circuit data into IRP. The Data required by RP's circuits is input in "In_dat". Kinds of data are pictures, sound, and so on. "Mem_ref" informs the usable

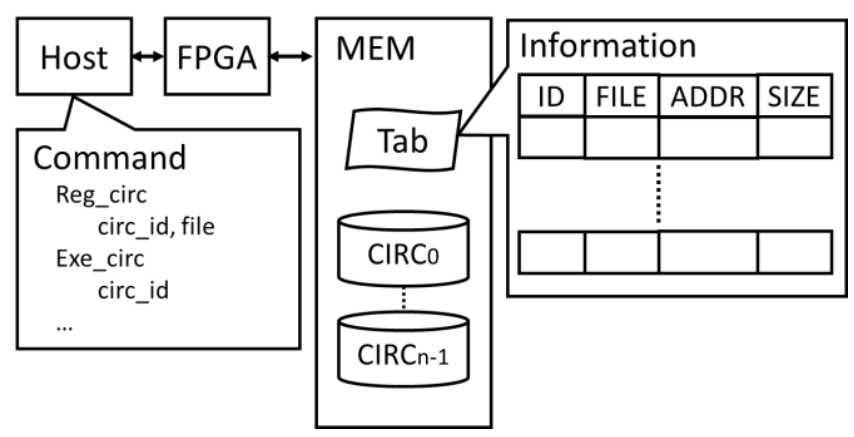

Fig. 4. The management framework of self DPR 
Table 1. Commands for management circuit data

\begin{tabular}{|l|c|c|}
\hline Command & Input & Function \\
\hline Reg_circ & ID, Circuit data & Registering circuit data \\
\hline Exe_circ & ID & Executing circuit data \\
\hline In_dat & Various & Inputting processing data \\
\hline Mem_ref & - & Referencing to usable storage \\
\hline Tab_ref & - & Referencing to tables \\
\hline
\end{tabular}

storage in a memory to users. In specific, this command gets ranges of the top and bottom address that users can operate without limitation. Referring to the table concerning circuit data registered in a memory is executed by the command of "Tab_ref". Users can execute self DPR by these functions without memorizing registered circuit data. And they can omit time for executing by the simplification of operations.

\section{Prototype System}

\subsection{Hardware Organization}

FPGAs using in this verification are ARTIX7 (XC7A35T-2CSG324C) and ZYNQ (XC7Z0101CLG400C) made by Xilinx. The former configure six RPs,

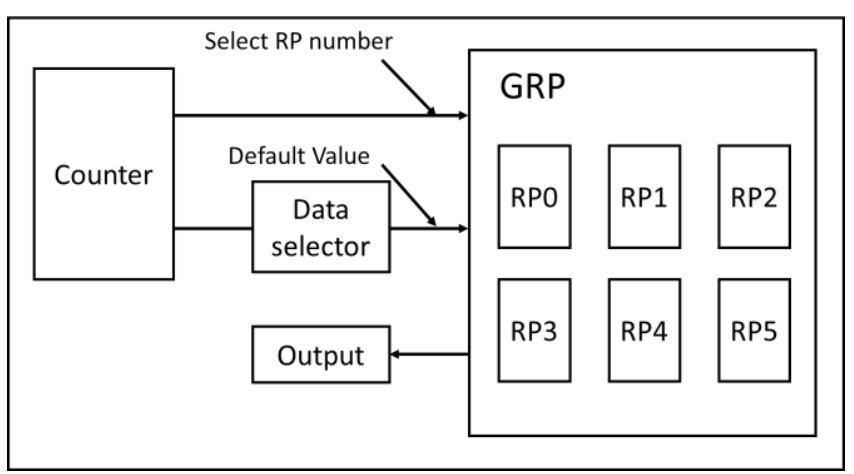

(a) Circuit outline

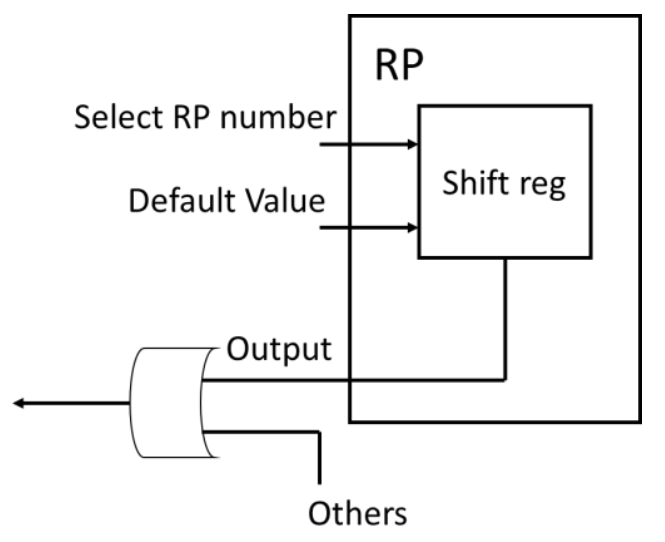

(b) RP contents

Fig. 5. Circuits of ARTIX-7

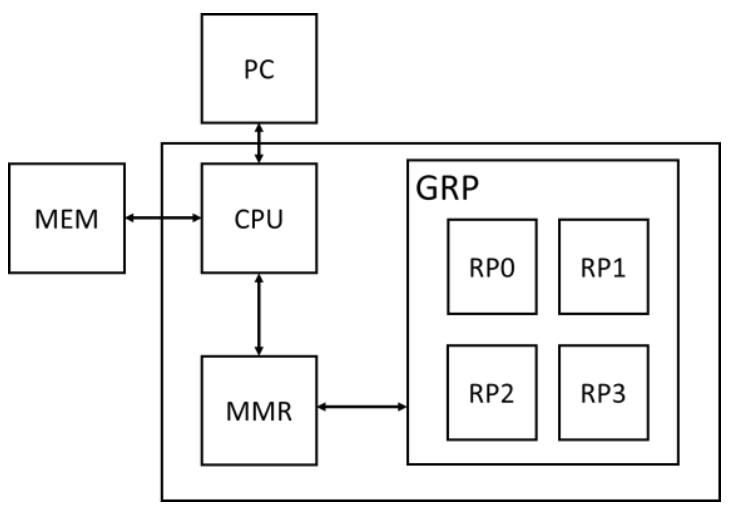

(a) Circuit outline

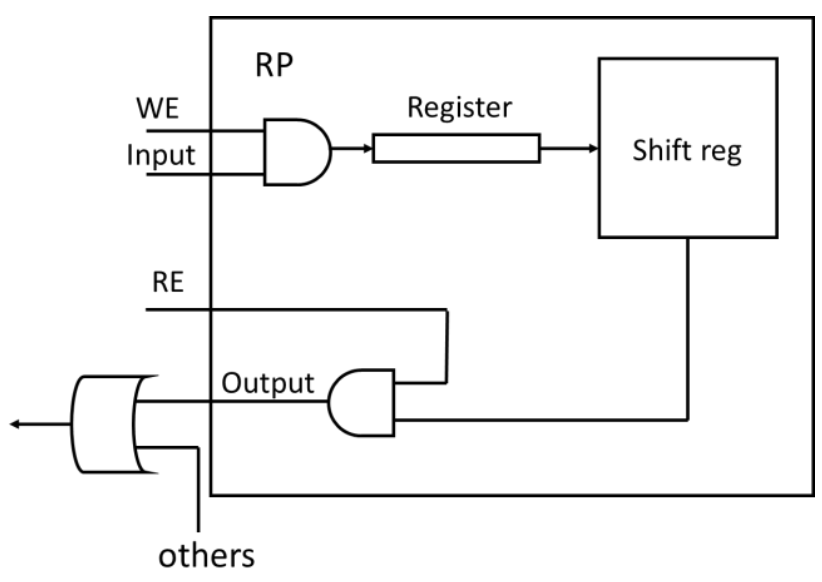

(b) RP contents

Fig. 6. Circuits of ZYNQ

the latter is four. Each RPs are implemented the shift register circuit occupying about $96 \%$ of internal resources.

(a) of Fig. 5 and 6 shows each circuits. (b) of them shows contents of each RPs. In case of simply executing only DPR, it is sufficient to design RPs and to prepare circuit data. But the RP executing the blanking must be in active because the purpose of this verification is power saving. For example if the shift register circuit of RPs has no default value, all bit internal the circuit are ' 0 '. The RP in this state isn't in active. So, the blanking has no meaning. Circuits are designed a default value is input with always changing from the outside of RPs because avoiding a disappearance of circuits by the optimization in designing.

Fig. 5(a) is the circuit implemented on the ARTIX-7. (b) is contents of RPs. The counter synchronizes with the internal clock and create numbers to input to the data selector circuit and select RPs inputting a default value. The data selector circuit outputs bit patterns corresponding to the number input from the counter. Thus each RPs become active because a default value of all RPs change with high speed. It means that measuring the effect of power saving by the blanking is possible. In fact Group of Reconfigurable 
ports (GRP) doesn't exist but it is indicated to help understanding this figure. As a result the GRP outputs the logical disjunction of the last registers of the each RP's circuits.

Fig. 6 (a) is the circuit implemented on the ZYNQ. (b) is contents of RPs. In this circuit, each RPs have registers for inputting a default value. RPs become active by inputting a default value. Then the effect of power saving using the circuit blanking is measured by implementing circuit data for the blanking. This is an outline of operations. Memory Mapped Register (MMR) in this figure is the processing part judging input information from the CPU. Write Enable (WE) is the signal permitting to write. It is ' 1 ' when a default value is input to the register. A value of the register doesn't renewal when it is ' 0 '. Read Enable (RE) is the signal permitting to read. It is ' 1 ' when the last register outputs a value. An outputting value is ' 0 ' when it is ' 0 '.

\subsection{Software Organization}

The verification using the ZYNQ was carried out using the trial system conform to the management framework in the chapter 3. Fig. 7 is an outline of the trial system. The trial system has five functions, "Registration", "Implementation", "LED check", "Input or output" and "Memory reference". "Registration" carries out registering circuit data to the memory, "Implementation" is reading and executing. These functions are necessary for self DPR.

The flowchart of "Registration" is Fig. 8. Solid lines in this flow indicate the processing by inputting of users. Broken lines indicate the processing with executed automatic between the PC and the embedded processor. Users input the ID and circuit data and they are sent to the embedded processor. The PC gets file size of circuit data and send to the embedded processor when the message of completion is

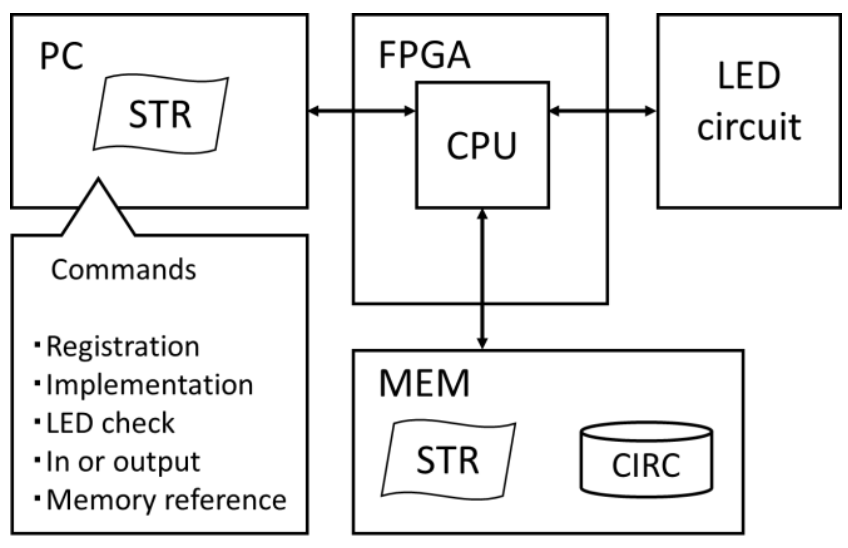

Fig. 7. Trial system outline



Fig. 8. Flowchart of "Registration"

sent from the embedded processor to the PC. File size is resent from the embedded processor to assess whether contents of file size sent by the PC are right. Then circuit data is sent from the PC to the embedded processor. The embedded processor gets the top address of circuit data registered on the memory. At the last, each side return to the idle state after writing to structures.

Fig. 9 is the flowchart of "Implementation". In this phase users only input information of the ID. The embedded processor looks for the ID coincide with in the structure when it received the ID. The DPR is executed writing circuit data with the corresponding ID to the IRP. The embedded processor sends the response to the $\mathrm{PC}$ and return to the idle state. The PC returns to the idle state receiving the response.

"LED check" is one of commands in this system makes 8-bit LEDs light implemented for outside a FPGA. These LEDs play a role by changing lighted bit in case of designing software or in each phase like registering or executing of circuit data. Fig. 10 indicates the flowchart of "LED check".

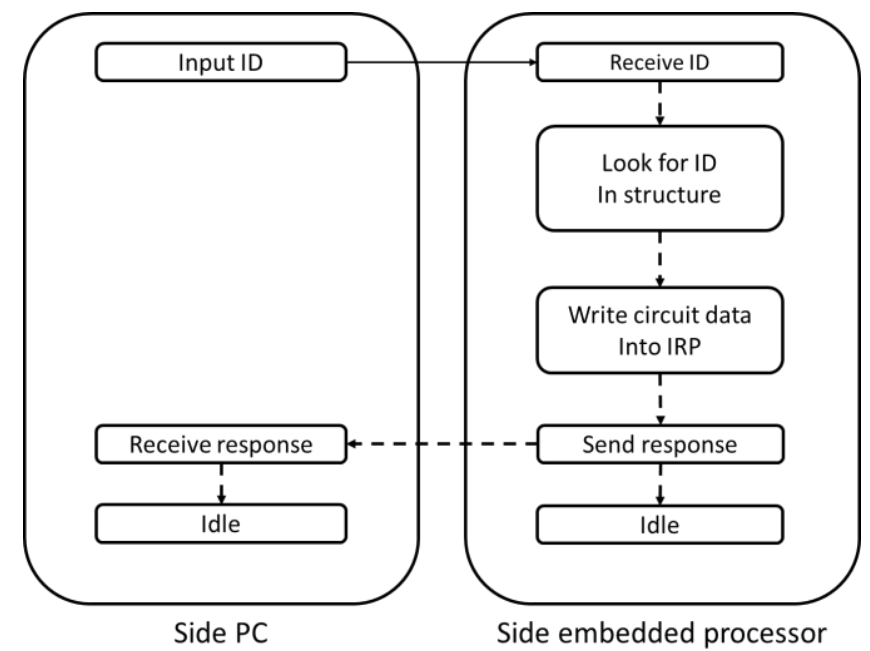

Fig. 9. Flowchart of "Implementation" 


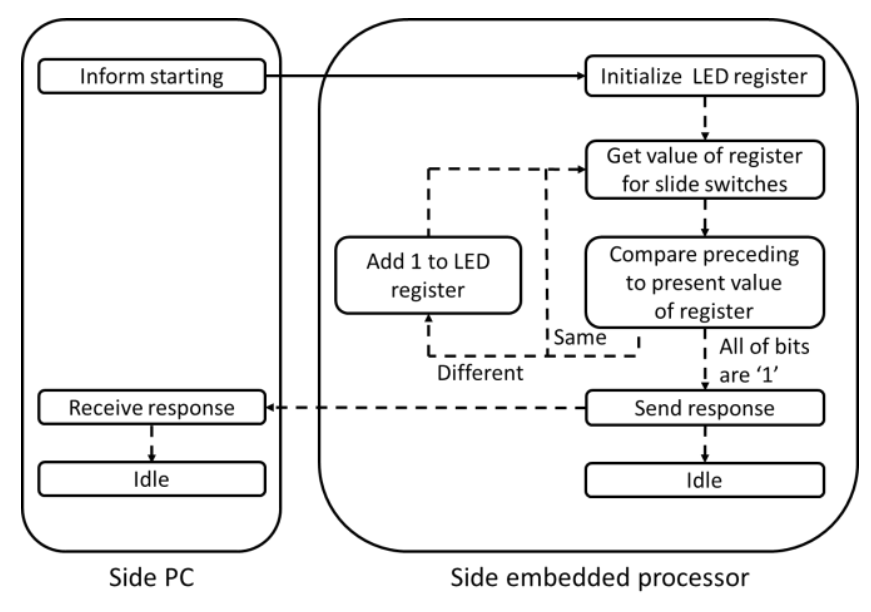

Fig. 10. Flowchart of "LED check"

The embedded processor initializes the register for LED when it is informed starting from the PC. It gets the value of the register for outside slide switch of the FPGA and compares preceding to present value of register. If two values accord, the embedded processor doesn't nothing and only repeat. The register is added 1 when they are different. In this state, the embedded processor ends processing if all of bits

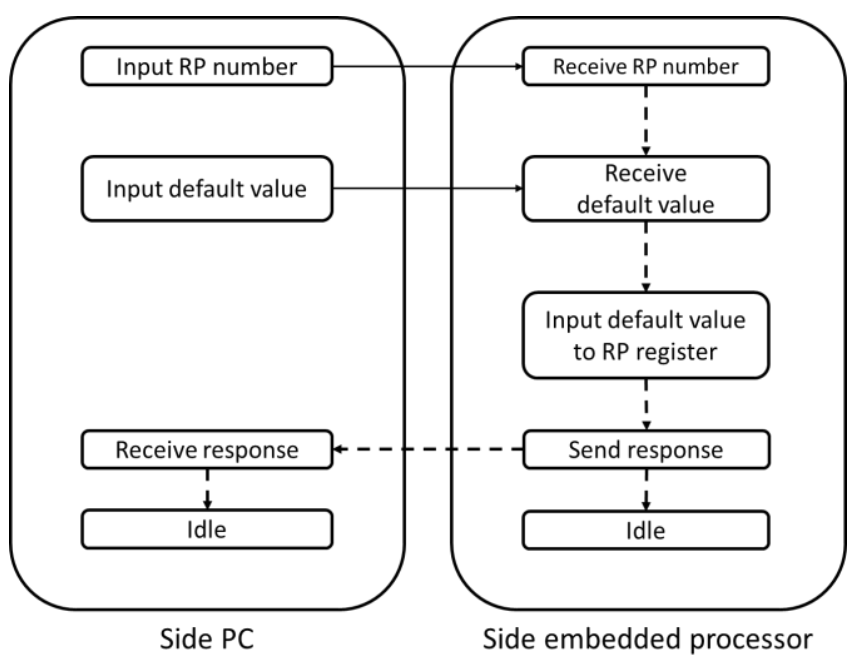

(a) Flowchart of input

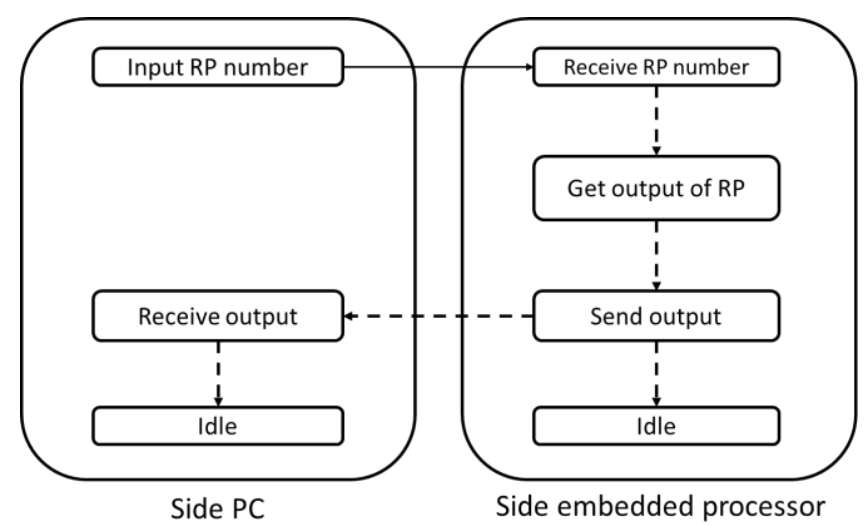

(b) Flowchart of output

Fig. 11. Flowchart of "Input or output"
Table. 2. Output table

\begin{tabular}{|c|c|c|c|c|}
\hline Number & ID & Filename & Size(bit) & Address \\
\hline 1 & 10 & RPO_blank.bin & 180572 & ffffcb90 \\
\hline$\vdots$ & $\vdots$ & $\vdots$ & $\vdots$ & $\vdots$ \\
\hline$\vdots$ & $\vdots$ & $\vdots$ & $\vdots$ & $\vdots$ \\
\hline
\end{tabular}

in the register for slide switches are ' 1 '. Then it sends the response to the PC and returns to the idle state. The PC returns to the idle state by receiving the response.

Inputting a default value and outputting a register in each RPs are executed by using "Input or output". Fig. 11 (a) is the flowchart of inputting. (b) is concerning outputting. In the figure (a), users input the default value and RP number. The embedded processor inputs the default value gotten by the PC to the register of the designated RP. It becomes the idle state after finishing inputting of the value and sending the response. The PC becomes the idle state after receiving the response. In the figure 11 (b), users input the number of RP that they want to be output to the PC. The embedded processor reads the last register in the corresponding RP, sends to the PC and returns to the idle state. The PC is returned to the idle state by receiving the response.

"Memory reference" outputs information of the memory. The top address is sent to the PC when users execute this command. Information of circuit data registered by "Registration" is preserved using the structure both the PC and the embedded processor as indicated Fig. 7. Users refer to only the structure of the $\mathrm{PC}$ in case of displaying information of circuit data. But we designed it to the embedded processor as well considering a potential of extension in future. The example of contents outputted by this command is the table 2. IDs and file names are necessary because users assess and manage circuit data registered the memory. Sizes and top addresses is gotten and displayed to visualize information of the memory.

\section{Experiment}

\subsection{Experimental Setup}

We measured the effect of power saving based on hardware and software indicated in the chapter 4. Fig. 12 is the circuit for measurement. The FPGA is connected like this figure and circuit data are loaded all RPs of the FPGA. The way executing blanking in each FPGAs is different. In the ARTIX-7, sending circuit data for circuit blanking to IRP from the PC makes RPs empty. We use the way that writing circuit data to IRP directly from PC because an ARTIX-7 has 


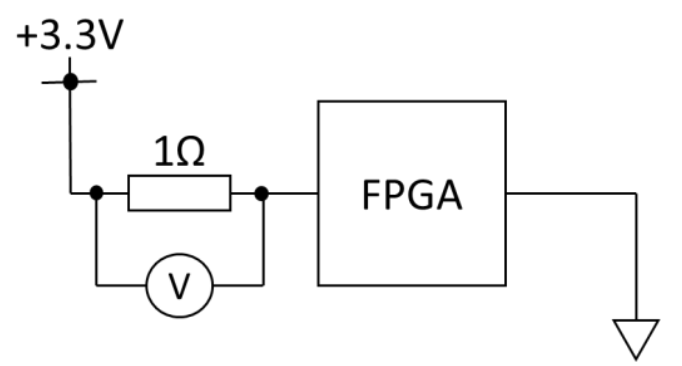

Fig. 12. Circuit for measurement

no embedded processors. The ZYNQ executes self DPR using trial system. Circuit data for circuit blanking is written to the memory and implemented from it. In each FPGAs, we measure voltage drop of the resistance connected outside of the FPGA on the occasion of circuit blanking. And we assess the effect of power saving by calculating a current value in the circuit.

\subsection{Result and Discussion}

Fig. 13 is verification result that current value is normalized as 1 (NCV) in case of that we doesn't execute a circuit blanking. Both of graphs indicate that current values decline by executing circuit blanking. We thought that current values decline because operation rate of nodes in RPs by executing circuit blanking goes down. And we can find that the ARTIX-7 is higher than the ZYNQ concerning the



(a) ARTIX-7

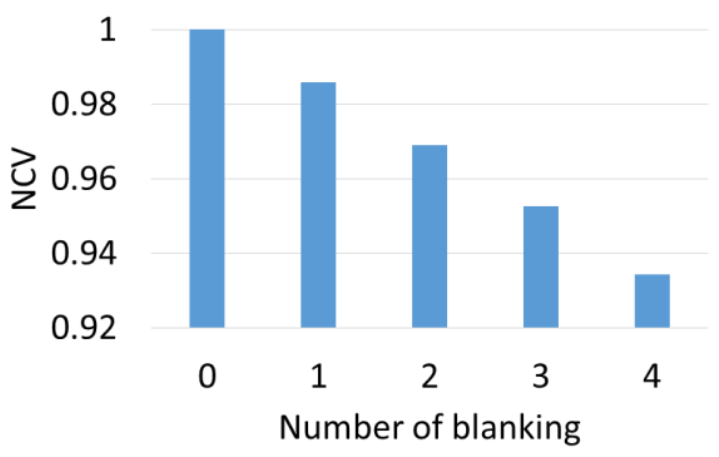

(b) ZYNQ

Fig. 13. The change of NCV by the RP blanking. effect of power saving. The reason is that the ARTIX-7 has no peripherals.

\section{Conclusion}

In this research, we defined the framework of the design and management methods for the self DPR. Then, we assessed the effect of power saving by circuit blanking using the trial system. As the result of this experiment, circuit blanking was confirmed that it is in effect for power saving and the effect is big in case of using no peripherals and only FPGA in particular. As further work, we will indicate the design automation and utility of power saving by circuit scale.

\section{References}

(1) Seyyed Mahdi Najmabadi, Zhe Wang, Yousef Baroud, and Sven Simon : “Online Bandwidth Reduction Using Dynamic Partial Reconfiguration", Proceedings of the $24^{\text {th }}$ IEEE International Symposium on FieldProgrammable Custom Computing Machines, pp. 168171,2016

(2) Gianluca Durelli, Fabrizio Spada, Riccardo Cattaneo, Christian Pilato, Danilo Pau, and Marco D. Santambrogio : “Adaptive Raytracing Implementation using Partial Dynamic Reconfiguration”, Proceedings of 2014 IEEE 28 ${ }^{\text {th }}$ International Parallel \& Distributed Processing Symposium Workshops, pp. 236-242, 2014

(3) Gayathri R Prabhu, Bibin Johnson, and Sheeba Rani J : "FPGA based Scalable Fixed Point QRD core using Dynamic Partial Reconfiguration”, Proceedings of 2015 $28^{\text {th }}$ International Conference on VLSI Design and 2015 $14^{\text {th }}$ International Conference on Embedded Systems, pp. $345-350,2015$ 